\title{
Shoulder surgeon and autologous cellular regeneration - From bench to bed: Part one- the link between the human fibroblast, connective tissue disorders and shoulder
}

\section{Don F Du Toit}

Division of Anatomy and Histology, Academic Department of Biomedical Sciences, Faculty of Health Sciences, University of Stellenbosch; Diabetes Mellitus Discovery Research Platform, Medical Research Council, Parow, South Africa

Wayne G Kleintjes

Plastic and Reconstructive Surgeon, Suite 1008, Louis Leipoldt Hospital, Bellville, South Africa

Erick J Mazyala

Division of Anatomy and Histology, Department of Biomedical Sciences, Faculty of Health Sciences, University of Stellenbosch, South Africa Deepak N Bhatia

Cape Shoulder Institute, Suite 4, Medgroup House, Plattekloof, Cape Town, South Africa

Joe F De Beer

Cape Shoulder Institute, Suite 4, Medgroup House, Plattekloof, Cape Town, South Africa

Benedict J Page

Plastic and Reconstructive Surgeon, Suite 1008, Louis Leipoldt Hospital, Bellville, South Africa

\section{Abstract}

The process of autologous cell regeneration (ACR) is a facet of cell therapy and regenerative medicine. It is initiated when activated autologous platelet rich plasma alone (PRP), containing cytokines or growth factors, is injected into the dermis or other structure in order to initiate a regenerative or antiaging process. The recipients resident cells at the target zone are activated by biologically active growth factors, derived from the activated platelets in the PRP through a paracrine effect. The platelet gel that contains the fibrin and clumped platelets, releases growth factors that influences activation of macrophages and stem cells in the recipient site. Tissue regeneration is facilitated by stem cell proliferation and differentiation. The PRP that has sealant and wound healing properties, may shift the wound healing cascade to the left, thereby speeding up tissue regeneration and remodelling by the use of the recipients own plasma.

Both the fibroblast and myofibroblast play key roles in the wound healing cascade. The fibroblast, of mesenchymal origin, plays a pivotal role in the formation of the extracellular matrix and deposition of collagen. A failure of organised fibroblast function results in important and disabling disease processes and conditions such as chronic ischaemic heart disease and remodelling of the heart, lung fibrosis, fibromatosis, solar aged-face, keloids, hypertrophic scar formation, nodular fasciitis, inguinal hernia, Dupuytren's disease and scleroderma. More recent additions include frozen shoulder and the captured shoulder. In aesthetic medicine, one of the prime functions of fractional photothermolysis with lasers, intense pulsed light and radiofrequency devices, is to stimulate dermal fibroblast proliferation, by thermal energy thereby increasing collagen deposition which enhances facial rejuvenation. Platelet -rich plasma (REGENLAB PRP, REGENLAB-ACR) possesses unique growth factors that stimulate, fibroblast, keratinocyte and myoblasts ex vivo in tissue culture, allowing threedimensional cell proliferation within the fibrin gel. PRP can be used as a cell carrier (i.e., keratinocytes, fibroblasts), may enhance cell retention at the point of treatment. REGENKIT is authorized for human use, ISO and CE marked. In this overview fibroblast morphology, tissue culture and cell biology relevant to the shoulder surgeon is reviewed.

Keywords: AACR, adhesive capsulitis, captured shoulder, coracohumeral ligament, fibroblast, frozen shoulder, growth factors, light emitting diode, myofibroblast, photo light, photobiomodulation, platelet-rich plasma-platelet-derived factors, platelet rich plasma, regeneration, rejuvenation, rotator cuff repair, shoulder surgery 


\section{Introduction: Definitions and developmental aspects}

Gray's Anatomy, the definitive work on the structure and function of man, gives a vivid account of the connective tissue that is derived from the mesenchyme. ${ }^{[1]}$ Connective tissue consists of cells embedded in a matrix or ground substance. ${ }^{[1],[2],[3]}$ The ECM was previously referred to as the intercellular substance. ${ }^{[1]}$

Dorland's Illustrative Medical Dictionary defines a fibroblast as an elongated-shaped connective tissue cell with vesicular, oval nucleus and cytoplasmic processes at each end. ${ }^{[4]}$ The cell is of mesenchymal or mesoderm origin. A myofibroblast is referred to as an atypical fibroblast displaying features of a fibroblast and a smooth muscle cell. Compared to fibroblasts, a myofibroblast has an irregular nucleus, abundant RER in the cytocavitary network and numerous collections of myofilaments. ${ }^{[4]}$ The term mesoderm refers to cells derived from the epiblast and extraembryonic tissues. ${ }^{[5]}$ In Langman's Medical Embryology, the term mesenchyme is used and refers to loosely organised embryonic connective tissue regardless of origin. ${ }^{[5]}$ In the embryo, the process of gastrulation leads to the development of the three germ layers (ectoderm, mesoderm and endoderm) in the third week of gestation. ${ }^{[5]}$ For the purposes of this review then, undifferentiated pluripotent mesenchymal cells can give origin to either fibroblasts or lipoblasts. ${ }^{[3]}$ The latter cell line develops into uni or multilocular adipocytes. Multilocular (or brown) adipose tissue is comprised of numerous lipid droplets and abundant brown mitochondria. ${ }^{[3]}$

An understanding of the morphology of the fibroblast and myofibroblast is important as these cells play an important function in the formation of the ECM and collagen deposition, relevant to wound healing [Figure - 1]. Active cellular regeneration (ACR) refers to dermal or tissue regeneration/rejuvenation therapy via autologous cell rejuvenation. Central to this theme, is the use of PRP which contains important growth factors that favorably impacts on the wound healing cascade [Figure - 2]. REGENLAB in Switzerland coined the term REGENACR, thereby implying volumetric and cell regeneration, platelet and leukocyte release of growth factors, stem cell proliferation and differentiation. The purpose being to facilitate the biological process of tissue regeneration and remodelling. For this purpose PRP can be used alone or in combination with cells such as keratinocytes, fibroblasts, myoblasts, adipose-derived stem cells, limbal corneal stem cells, chondrocytes, pancreas islet cells, stem cells and Schwann cells [Figure - 3]. The platelet/fibrin gel then serves as a cell vehicle carrier or biological cell scaffold/construct. This approach via tissue engineering is aimed at enhanced cell retention (by enhancing cell embedding) at the recipient site of cell therapy. In other words, the donor cells that are engrafted as a platelet gel and cells or with nanotechnology for regenerative purposes (i.e. the skin with fibroblasts or myocardium with myoblast progenitor cells), will be retained at the recipient tissue bed and prevented from being washed away in the lymphatics and venules shortly after injection. The advantage is that autologous cell transport medium and cells are utilized and the problems of immunological rejection and transmission of disease is obviated. And tissue regeneration is addressed by delivering GF's (from the activated platelets) at the recipient site as well as new donor cells (i.e. keratinocytes, myoblasts, fibroblasts, fat cells, stem cells and Schwann cells, chondrocytes, islet and beta-cells for diabetes mellitus).

\section{Fibroblasts: Types and functions}

Classically, the fibroblast (denotes an active cell) and fibrocyte (quiescent cell) is described in histology textbooks. ${ }^{[3],[6]}$ Basic histology at light microscopic level is reflected in [Table - 1] and [Figure - 4]. From a cell biology point of view, fibroblasts grow actively in monolayer culture, while fibrocytes show stagnant growth potential [Figure - 5]. This is important as regards the regenerative 
capacity of the connective tissue. The functions of fibroblasts are well documented and reflected as follows. ${ }^{[4],[6]}$

- Synthesis of proteins, collagen and elastin (collagen types I, II, III, V, XI). ${ }^{[4]}$

- Synthesis of reticular and elastic fibers, glycosaminoglycans, proteoglycans and glycoproteins of the extracellular matrix (ECM).

- Production of growth factors (influence cell growth and differentiation)

- Transformation into myofibroblasts during wound healing (demonstrates features of both fibroblasts and smooth muscle; contain actin microfilaments and myosin; responsible for wound contraction during wound healing and closure). The active secretory fibroblast cytoplasm is more apparent because of the presence of RER.

- Production of collagen is reflected in [Table - 2] and pathological conditions related to fibroblast dysfunction are reflected in [Table - 3].

- $\quad$ Release of serotonin, interleukin and prostaglandins

The cytoplasmic markers of human fibroblasts are as follows:

- Monoclonal antibody, ER-TR7 (expressed by reticular fibroblasts)

- Fibroblast- specific protein (FSP-1)

- 5BS antibody (FBS prolyl 4-hydroxylase)

- Antibodies are available for the identification of collagen, types I, II, III.

- Antibodies to actin and vimentin are useful for the identification of myofibroblasts

\section{Clinical disorders associated with dysfunctional collagen synthesis and metabolism : After Junqueira}

- Ehlers-Danlos type IV (Faulty transcription: inherited defect in procollagen peptides)

- $\quad$ Ehlers-Danlos type VI (Faulty lysine hydroxylation)

- $\quad$ Ehlers -Danlos type VII (decreased procollagen peptidase activity)

- $\quad$ Scurvy (Vitamin C deficiency: cofactor for proline hydroxylase)

- Ostegenesis imperfecte (gene defect affecting collagen type I)

- Marfan's syndrome is an autosomal dominant disease in which elastic tissue is weakened. Three systems are affected, ocular, skeletal and cardiovascular. Deficiency in the protein fibrillin I is responsible for the syndrome.

\section{Myofibroblasts: Relevance to wound healing orthopaedic surgery and disease}

It is now established that the myofibroblast can be found in normal tissue as well as in a wide variety of pathological processes. ${ }^{[7],[8],[9],[10],[11]}$ Myofibroblasts stain positively for alpha-smooth-muscle-actin, fibronectin, vimentin and occasionally desmin [Figure - 6]. ${ }^{[9]}$ Disease processes in which myofibroblasts feature strongly include, hypertrophic scar, Dupuytren's palmar fibrosis, nodular faciitis, the fibromatosis, keloids, postoperative spindle-cell nodule, fibroma of the tendon sheath, myofibroblastoma and inflammatory myofibroblastic tumors. ${ }^{[9]}$ Important aspects, regarding myofibroblasts need emphasizing and are enumerated below:

- Cultured myofibroblasts, like dermal fibroblasts can secrete type I and III procollagens into culture media. ${ }^{[10]}$

- $\quad$ Cultured myofibroblasts as well as dermal fibroblasts can be stained with antibodies directed at type I and III procollagens. Therefore, fixed cells can be accurately examined by 
immunohistochemistry. ${ }^{[10]}$ By electron microscopy, it is possible to demonstrate that myofibroblasts portray well-developed dense microfilaments of 40-80 degrees and that are prominent in the long axis of the cells. ${ }^{[10]}$ Some researchers view the myofibroblast as a modified fibroblast, following differentiation in response to specific signals from the extracellular matrix (ECM). ${ }^{[10]}$

- Myofibroblasts play an integral part in the wound healing cascade, wound contraction and hypertrophic scar formation during the healing of wounds. ${ }^{[11]}$

- Myofibroblasts appear in the initial phase of wound healing, generate contractile forces to pull both edges of an open wound together and with time undergo apoptosis. ${ }^{[11]}$ If apoptosis is disorderly, myofibroblasts have an increased propensity to form wound contracture of the hypertrophic scar. ${ }^{[11]}$ Myofibroblasts also play an important role in shoulder adhesive capsulitis. Other workers have shown that myofibroblasts play an important role in formation of granulation tissue and fibrocontractive diseases. ${ }^{[12]}$ If myofibroblasts become overactive and continue, then there is accumulation of excessive amounts of ECM. ${ }^{[12]}$

- Myofibroblasts can secrete inflammatory and anti-inflammatory cytokines, growth factors, lipid and gaseous inflammatory mediators as well as extracellular matrix proteins and proteases. ${ }^{[13]}$ Therefore myofibroblasts play an important role in organogenesis, oncogenesis, inflammation, repair and fibrosis in tissues. ${ }^{[13]}$ Workers at the University of Texas Medical Branch at Galveston, have shown that transforming growth-factor-beta and the PDGF family are key players in the fibrotic response. ${ }^{[13]}$

- Lung fibrotic remodelling, as seen in pulmonary fibrosis is a feature of fibroblast/myofibroblast activation that results in progressive organ dysfunction, usually with a terminal outcome. ${ }^{[14]}$ Possibly, the activation of the myofibroblasts is by a shift in the so called T-helper 2 type cytokine network. ${ }^{[14]}$

- Granulation tissue fibroblasts and myofibroblasts express alpha-sm actin. Apparently, when granulation tissue evolves into a scar, myofibroblasts containing-sm actin disappears due to apoptosis. ${ }^{[15]}$ Myofibroblasts develop tensile force through the neoformation of alphasmooth muscle actin containing cytoplasmic stress fibers. ${ }^{[16]}$ This generation of wound tension has been shown to be a crucial regulator of connective tissue remodelling. ${ }^{[16]}$ Also, cell-cell adherens junctions appear to synchronise myofibroblast contractile activity. ${ }^{[16]}$ Clearly then, myofibroblasts are necessary for mechanical force development. Researchers from Bordeaux, France have shown that fibroblast/myofibroblast transition begins with the appearance of the protomyofibroblast, whose stress fibers contain only beta-and-gammacytoplasmic actins. ${ }^{[17]}$ With time, the differentiated myofibroblast displaying stress fibers and alpha-smooth muscle actin becomes evident. ${ }^{[17]}$

- The unique capabilities of the myofibroblast to induce production of tension in wounds, relevant to wound healing and pathological organ remodelling has been established. ${ }^{[18]}$

- Histological features of myofibroblasts include a plump-spindle-cell morphology, ill defined cytoplasm and less fibrillar than smooth-muscle cells and matrix collagen. ${ }^{[9],[19]} \mathrm{A}$ histological distinction can be made between smooth muscle and myofibroblastic lesions by studying the cell surface that is characterized by prominent fibronectin fibrils and fibronexus junctions. ${ }^{[9]}$

- Other researchers, working independently have shown that myfibroblasts can be identified in several regions of the body, such as the skin, periodontal ligaments and pulmonary septa.

\section{Healing of surgical wounds by First intention: Role of the fibroblast locomotion}

The wound healing cascade, consisting of inflammation, granulation tissue, wound contraction, collagen accumulation and remodelling is well understood. ${ }^{[19]}$ By day three, collagen is deposited by fibroblasts and progresses steadily through the third week. ${ }^{[19]}$ The following factors inhibit wound healing:

- Vitamin C deficiency: inhibition of collagen synthesis and fibroblast proliferation 
- Glucocorticosteroids: diminished fibrosis

- Ischemia

- Nutritional disorders (kwashiorkor and marasmus)

- Excessive production of collagen (keloids) and granulation tissue (i.e. in burns). Immune and autoimmune reactions

\section{Cell biology: Fibroblast tissue culture, dynamics, biostimulation with Platelet-derived growth factors (PRP) and Light-Emitting-Diode (LED) photolight therapy}

Culture of the skin ex vivo by explant technology is a well-established practice in dermatology. ${ }^{[20]}$ In our cell biology facility at Stellenbosch, we have been able to document the following laboratory observations regarding dermal fibroblasts culture ex vivo with varying concentrations of platelet-rich plasma (REGEN-PRP) and other conventional growth media. Dermal fibroblast outgrowth and proliferation is as follows.

- Human fibroblasts in tissue culture (TC) take about five to seven days to appear from explants and cultured at the air-interface ("air-lifting") with or without serum enhanced or serum starved media. On fibronectin enriched plates, monolayers are generated but cell growth is $2 \mathrm{D}$ or planar.

- Cell yields and proliferation is enhanced by medium enrichment with PRP ex vivo compared to controls and serum starved medium. This follows by the stimulation of PDGF, TGF and allows cell expansion and proliferation in 3D and monolayers [Figure - 7].

- REGEN PRP enhances fibroblast locomotion, spreading by the activation of filapodia and lamellipodium. REGENKIT-PRP cell proliferation is greater than other extracts of PRP. Cell ruffling is evident. Collagen production is enhanced in vitro by PRP. REGENPRP contains quantified potent trophic growth factors such as VEGF, PDGF, IGF and EGF. It is postulated that fibroblast proliferation is stimulated by epidermal stem cells cited at the dermalepidermal junction. REGENPRP is known to induce chemoattraction of stem cells, neutrophils and macrophages, proliferation as well as differentiation. With time, differentiation of stem cells occurs, allowing the remodelling of tissues and cells to occur. PDGF is known to be operational in the inflammatory phase of the wound healing cascade. In the regeneration phase. TGBF, EGF, IGF are also operational but some overlap does occur.

- Details on the biological activity of PRP are reviewed in the literature. ${ }^{[21],[22],[23],[24]}$ Plateletpoor plasma (PPP) and platelet rich plasma (PRP) can easily be separated from the plasma in a side room or theatre, but very careful centrifugation is indicated. ${ }^{[21],[22],[23]}$ PPP as well as PRP have sealant properties and PRP also has important biological wound healing activities. If the platelets in a blood clot are activated, by either calcium chloride or thrombin, release of important growth factors (GF) occurs. Important GF that impact on the wound healing cascade include, PDGF, TGBF alpha and beta, IGF and EGF. ${ }^{[21],[22]}$ The GF's or cytokines facilitate the process of wound healing, chemoattraction of fibroblasts and myofibroblasts, formation of ECM or scaffold and deposition of GAG and collagen. ${ }^{[21],[22]}$

- Of importance is the fact that diploid fibroblasts in cell culture undergo a finite number of divisions. ${ }^{[20]}$ At the present time we are not sure if PRP can reverse this important phenomenon. Work has been done and it has been demonstrated that senescent dermal fibroblasts over express metalloproteinase and theoretically explain age-related atrophy of ECM architecture. Because fibroblasts have a restrictive capacity of cell doublings, the cells eventually become unresponsive to growth factor stimulation. It is tempting to speculate that, PRP could at least ameliorate this restriction in ex vivo culture.

- Keratinocytes, through release of epidermal cytokines, can modulate their own phenotype, through autocrine pathways but also influence dermal fibroblast phenotype by paracrine mechanisms. Abnormalities in fibroblast function, such as the disturbed release of IL-6, play a 
central role in the pathogenesis of scleroderma. TGB-alpha is capable of upregulating type VII collagen gene expression in cultured dermal fibroblast and epidermal keratinocytes and this is valuable information when evaluating the biostimulatory and physiological effects of PRP.

- In our light laboratory we have been able to biostimulate and increase proliferation of fibroblasts ex vivo by the use of $633 \mathrm{~nm}$ non-thermal light source $\left(O \mathrm{mnilux} \mathrm{x}^{\mathrm{TM}}\right) .{ }^{[25]}$ We can thus manipulate and increase cell proliferation by two separate stimulation modalities (nonthermal light and REGENLAB-PRP which provides 7 secretary proteins, cytokines and growth factors), without a noticeable increase in apoptosis. However, the fluency and duration of light stimulation has to be adapted for in vitro work and cell proliferation and therefore differs from light intensity needed in the clinic for rejuvenation and anti-inflammatory purposes. The $633 \mathrm{~nm}$ light source provides unique features for cell stimulation and has the advantage of being athermal and not cell ablative as seen after application of low level laser energy stimulation [Figure - 8]. Nominal wave-length of 630-670nm (Omnilux $\left.{ }^{\mathrm{TM}}\right)$ can selectively stimulate fibroblasts and keratinocytes. However, $830 \mathrm{~nm}$ can super-selectively stimulate mast cells, neutrophils, macrophages, myofibroblasts and keratinocytes. A combination of these two light sources provides stimulation of mast cells, neutrophils, macrophages, fibroblasts, myofibroblasts and keratinocytes. LED (red light) is absorbed in the mitochondria of cells and leads to ATP stimulation. This may well result in increased cellular action potential and enhanced cell vitality. The $633 \mathrm{~nm}$ light (Omnilux $\left.{ }^{\mathrm{TM}}\right)$ emitted is potentially absorbed by fibroblasts and this will enhance the speed of neo-collagen synthesis. By $830 \mathrm{~nm}$ stimulation, myofibroblasts are activated and this will improve alignment of newly produced collagen bundles. The unique, non-thermal light source, is safe and applicable as an antiinflammatory device (i.e. in combination with lasers) and the treatment of acne and skin cancers. We provide strong research evidence for the application in facial rejuvenation (i.e. for the chronological and photo aged face with wrinkles and skin sagging). ${ }^{[25]}$

We confirm the observations of Albert's et al. , and have observed in our laboratory that fibroblasts (of mesenchymal origin and possessing totipotential characteristics) can be stimulated to change their character in tissue culture [Figure - 9]. ${ }^{[26]}$ We have observed that human fibroblasts do change shape and position in vitro and assume more complex process bearing morphologies when cultured in REGEN PRP. ${ }^{[26]}$ We speculate that the growth factors released by activated platelets in PRP, act as powerful regulators of growth, differentiation and matrix synthesis of connective tissue cells such as fibroblasts. ${ }^{\left[{ }^{26]}\right]}$ We agree with Albert's et al. that TGBF-beta (released by activated platelets in REGEN PRP) is important in wound healing and facilitates conversion of fibroblasts into myofibroblasts and thereby promotes the strength of wounds by deposition of collagen-rich scar tissue. ${ }^{[26]}$

Non-thermal light skin rejuvenation has the advantage over conventional lasers that produce similar results but at the cost of longterm scarification of the dermis. At this point in time, it is not known if fractional, non-ablative photothermolysis, will also be associated with scarification. Important work from the University of Johannesburg, showed that diabetic fibroblast cells at fluencies of 5 and 16 $\mathrm{J} / \mathrm{cm}^{2}$, in an experimental model, could favourably enhance cell migration, by the application of helium-neon laser irradiation. ${ }^{[27],[28],[29]}$ Also, they showed in this fibroblast model that affluence of 5 $\mathrm{J} / \mathrm{cm}^{2}$, stimulated mitochondrial activity and therefore acted as a modifier of cell processes. ${ }^{[27],[28],[29]}$ Optimizing correct energy density (fluency) and number of exposures is obviously important to ensure efficient fibroblast cell migration and proliferation, both in vivo and in vitro .

\section{Frozen shoulder: Associated with Dupuytren's disease}


Although young females between the ages of 40 and 55 years with a frozen shoulder recover almost totally, there have been studies showing that some have ongoing discomfort and limitation of external rotation. ${ }^{[30],[31],[32],[33]}$ In the orthopedic literature, a reference is made that frozen shoulder is an entity presenting with chronic fibrosis of the shoulder joint capsule. ${ }^{[32],[33]}$ Cellular infiltrate has been identified to consist of fibroblasts and myofibroblasts. A dense layer of type-III collagen matrix is deposited in the shoulder capsule. ${ }^{[30]}$ The collagen undergoes contraction resulting in pain and stiffness. The histological picture mimics those found in Dupuytren's disease. ${ }^{[30]}$ Recent literature shows that Dupuytren's disease is about 8 times more common in persons with frozen shoulder. ${ }^{[30]}$ Indeed, Schaer first noted the association between frozen shoulder and Dupuytren's disease in 1936. An association with diabetes mellitus and hyperlipidaemia has also been reported. Other workers report, in persons with the frozen shoulder syndrome, cytokine abnormalities and disorder of matrix metalloproteinase and metalloproteinase inhibitors. The two conditions seem to have very different natural histories. We agree that Dupuytren's disease is a progressive condition and frozen shoulder being a self-limiting disorder that fully resolves with time and have little long-term functional disability. The results of arthroscopic shoulder rotator cuff repair are excellent, with only a small percentage presenting with limited range of motion associated with persistent pain. This postoperative scenario of the "captured shoulder" is apparently caused by restrictive sub-deltoid adhesions and an associated an articular companion lesion, which no doubt is due to fibroblast stimulation and enhanced collagen deposition. ${ }^{[31]}$ These restrictive bands can be lysed at surgery but care is needed to avoid injuring the axillary nerve. In all these cases exclusion of unrecognized acromioclavicular disease and adhesive capsulitis is needed. Apparently, these patients benefit from re-intervention surgery and release of these adhesions results in decreased pain, increase in range of movement and return to near normal activities. Focused surgery in selected patients presenting with protracted symptoms of idiopathic adhesive capsulitis show deposition of scar tissue in the shoulder joint capsule. In addition there is thickening of the coracohumeral ligament. ${ }^{[30],[31],[32],[33]}$ Arthroscopic surgical release of the coracohumeral ligament and rotator interval can restore passive external rotation and relieves pain [Figure - 10] A and B.

\section{Rotator cuff repair and PRP augmentation}

Although young females between the ages of 40 and 55 years with a frozen shoulder recover almost totally, there have been studies showing that some have ongoing discomfort and limitation of external rotation. ${ }^{[30],[31],[32],[33]}$ In the orthopedic literature, a reference is made that frozen shoulder is an entity presenting with chronic fibrosis of the shoulder joint capsule. ${ }^{[32],[33]}$ Cellular infiltrate has been identified to consist of fibroblasts and myofibroblasts. A dense layer of type-III collagen matrix is deposited in the shoulder capsule. ${ }^{[30]}$ The collagen undergoes contraction resulting in pain and stiffness. The histological picture mimics those found in Dupuytren's disease. ${ }^{[30]}$ Recent literature shows that Dupuytren's disease is about 8 times more common in persons with frozen shoulder. ${ }^{[30]}$ Indeed, Schaer first noted the association between frozen shoulder and Dupuytren's disease in 1936. An association with diabetes mellitus and hyperlipidaemia has also been reported. Other workers report, in persons with the frozen shoulder syndrome, cytokine abnormalities and disorder of matrix metalloproteinase and metalloproteinase inhibitors. The two conditions seem to have very different natural histories. We agree that Dupuytren's disease is a progressive condition and frozen shoulder being a self-limiting disorder that fully resolves with time and have little long-term functional disability. The results of arthroscopic shoulder rotator cuff repair are excellent, with only a small percentage presenting with limited range of motion associated with persistent pain. This postoperative scenario of the "captured shoulder" is apparently caused by restrictive sub-deltoid adhesions and an associated an articular companion lesion, which no doubt is due to fibroblast stimulation and enhanced collagen deposition. ${ }^{[31]}$ These restrictive bands can be lysed at surgery but care is needed to avoid injuring the axillary nerve. In all these cases exclusion of unrecognized acromioclavicular disease and adhesive capsulitis is needed. Apparently, these patients benefit from re-intervention surgery and release of these adhesions results in decreased pain, increase in range of 
movement and return to near normal activities. Focused surgery in selected patients presenting with protracted symptoms of idiopathic adhesive capsulitis show deposition of scar tissue in the shoulder joint capsule. In addition there is thickening of the coracohumeral ligament. ${ }^{[30],[31],[32],[33]}$ Arthroscopic surgical release of the coracohumeral ligament and rotator interval can restore passive external rotation and relieves pain [Figure - 10] A and B.

\section{Acknowledgements}

Dr. Christo Muller (PHD) of The Medical Research Council, Parow, is thanked for manuscript review. Lionel Du Toit and Reginald Williams are thanked for technical assistance.

\section{Figures}

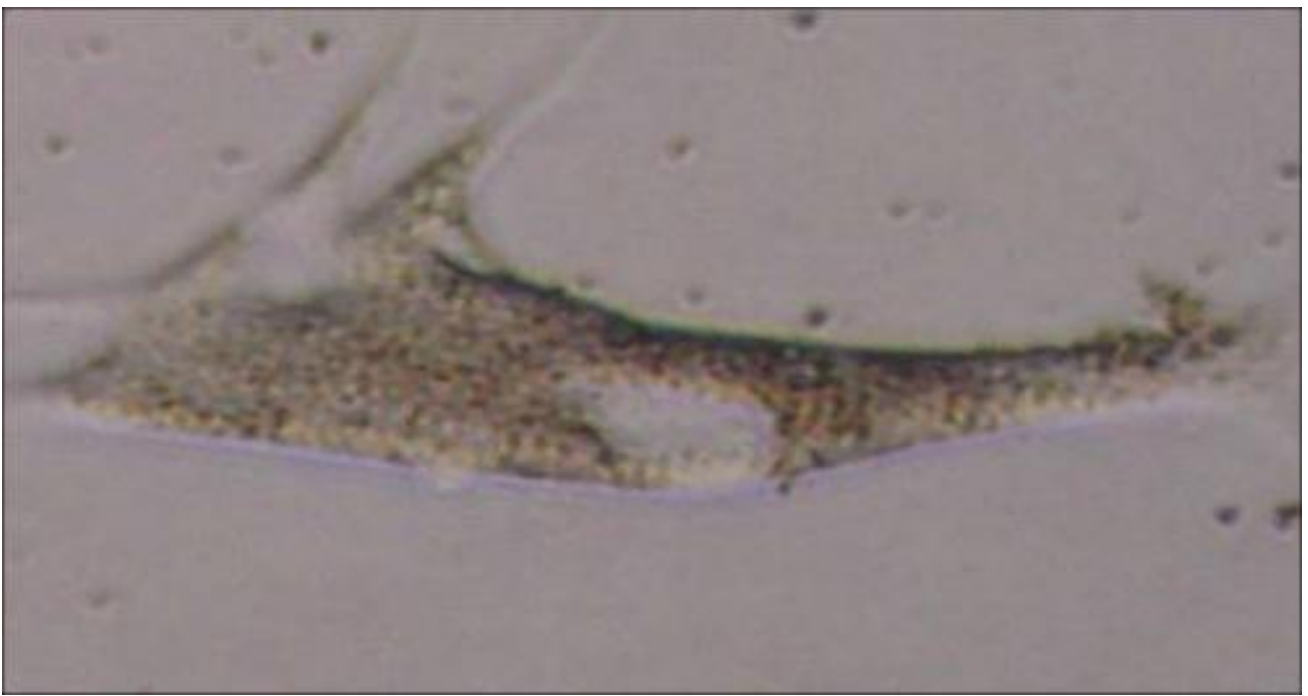

Figure 1: Morphology of human fi broblast expanded in REGENLABPRP, showing branching and filopodia x5000 (Olympus ${ }^{\circledR}$ inverted microscope). Other adjacent cells showed features of cell ruffling. Note nucleus, lamellopodium and RER 


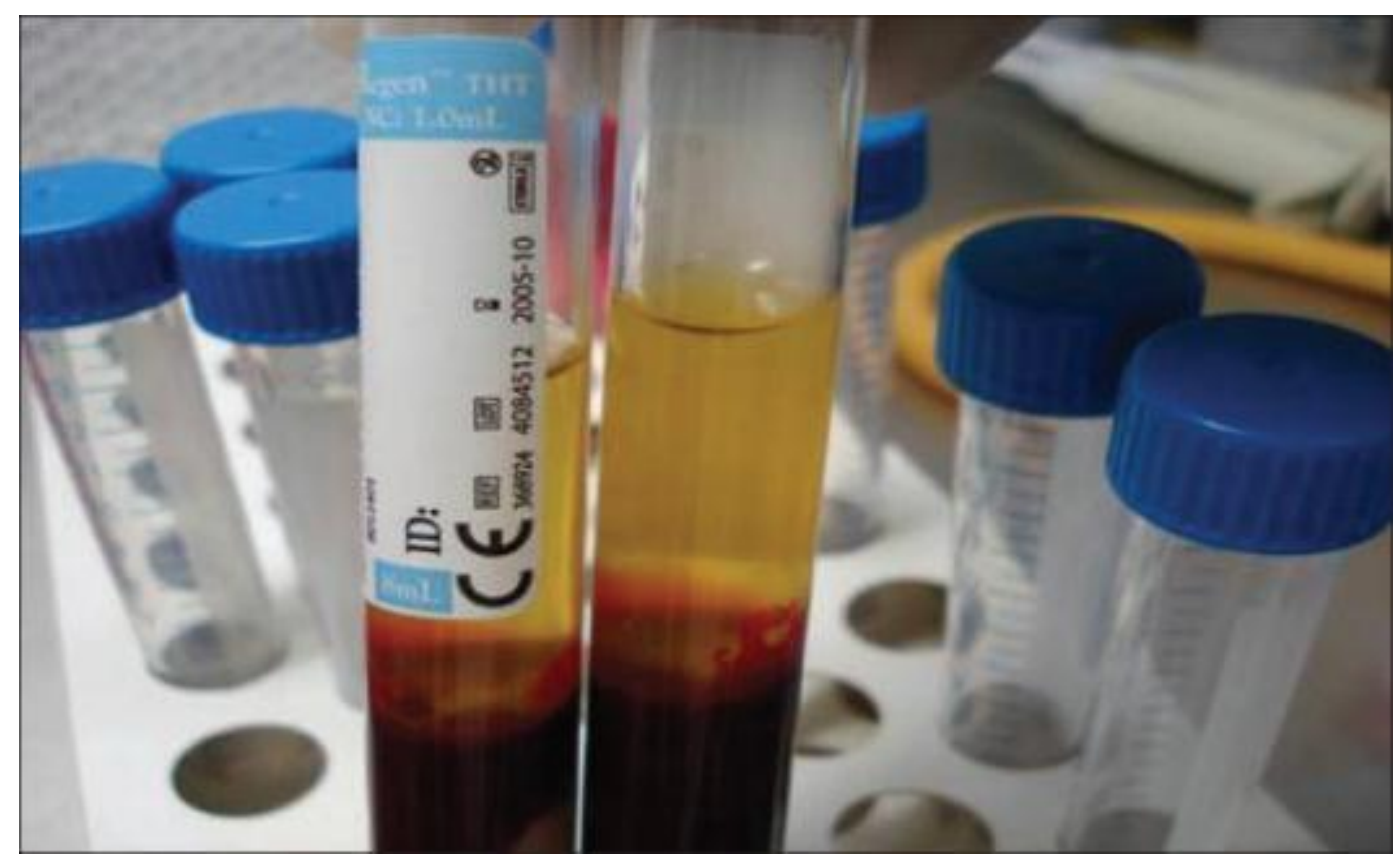

Figure 2: PRP is produced by centrifugation of anticoagulated venous blood taken from the medial cubital forearm vein. The centrifuge must be well-balanced and the buffy coat must be clearly visible. This is visible in the left tube. This layer contains neutrophils and platelets. Two spins may be necessary to obtain a good result

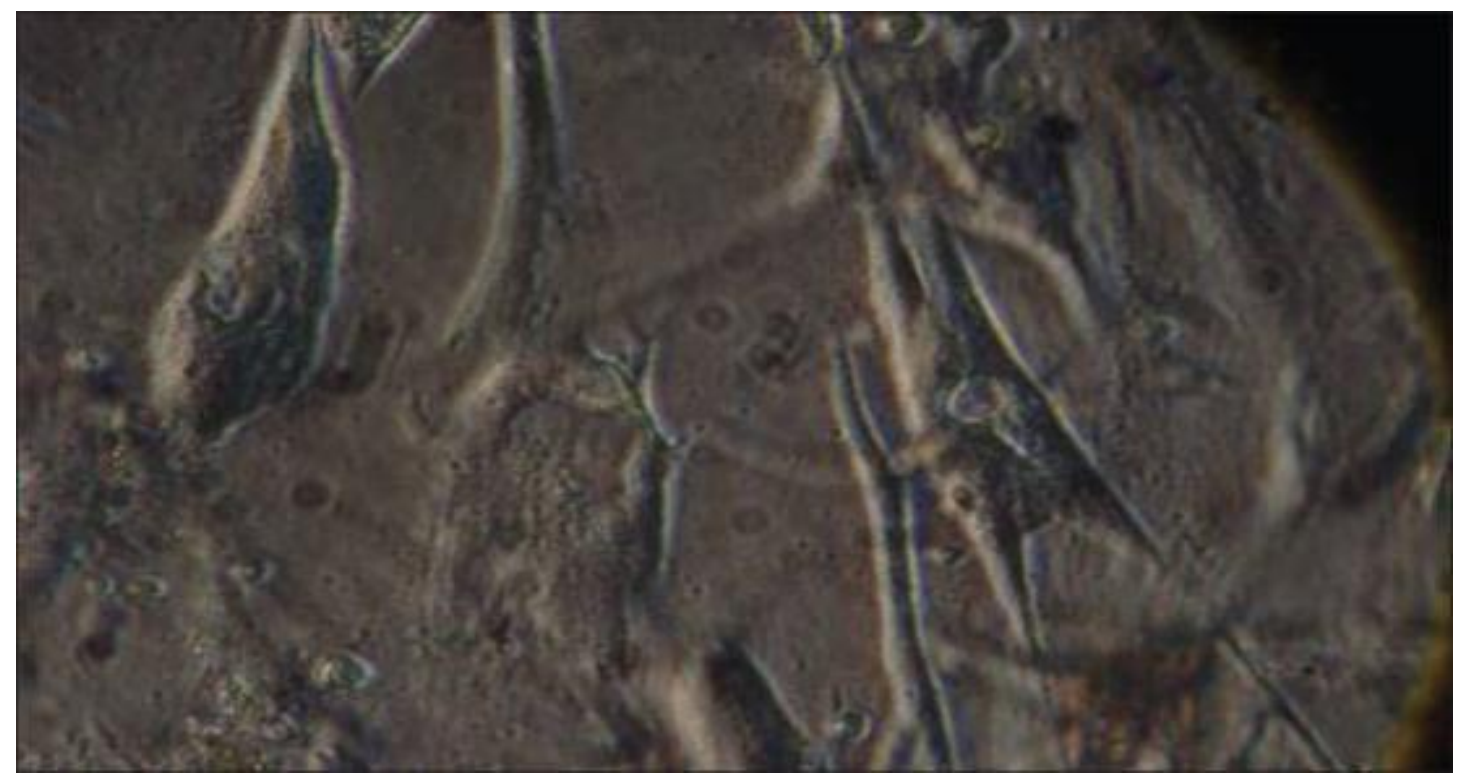

Figure 3: PRP primed primary cell culture and showing adipose derived-mesenchymal stem cells. Note adjacent apoptotic cell 


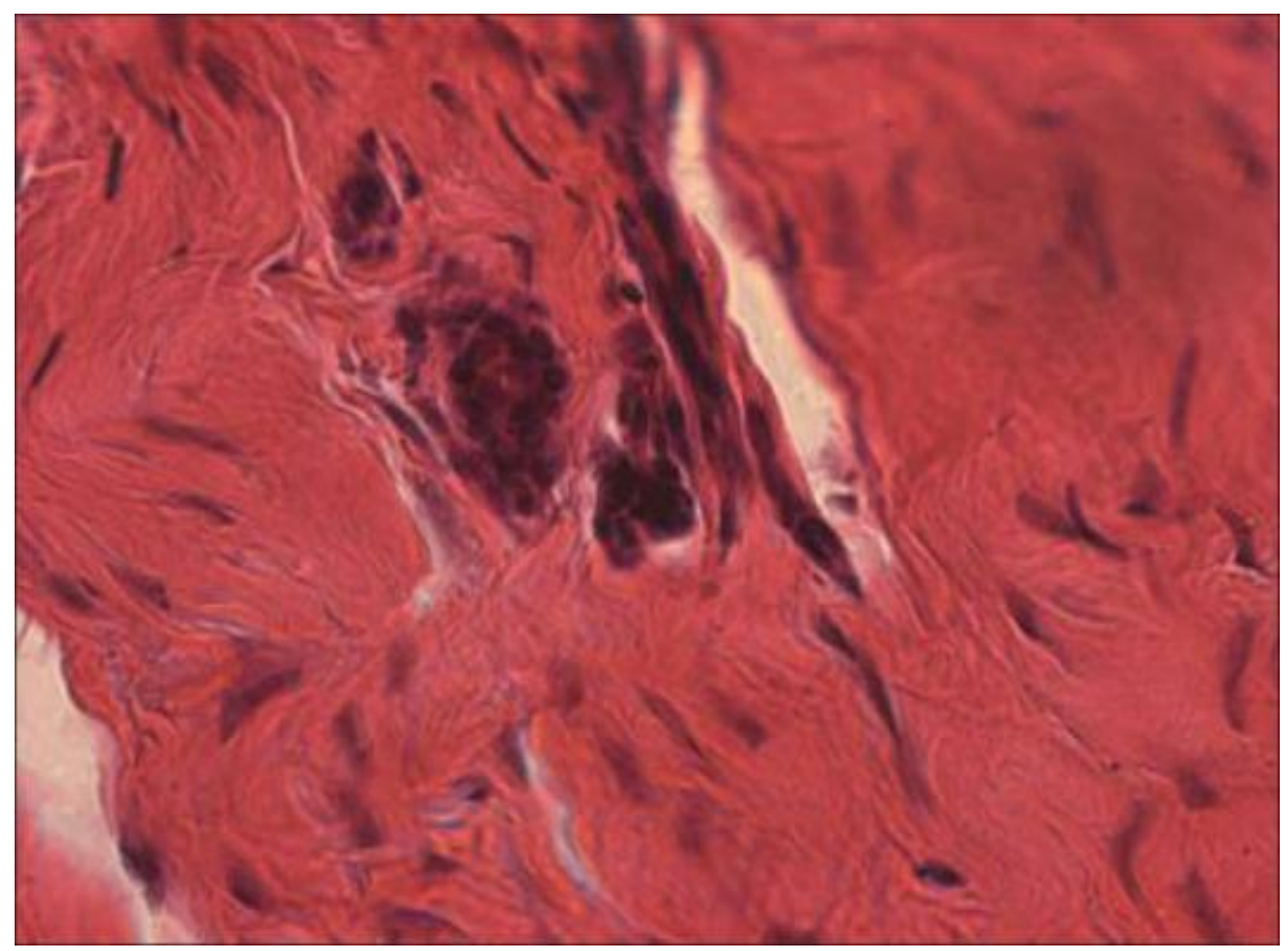

Figure 4: Light microscopy showing human fibroblasts in dense connective tissue ( $\mathrm{H}$ and $\mathrm{E}, \mathrm{X} 100)$ (With kind permission of Dr. W Kleintjes)

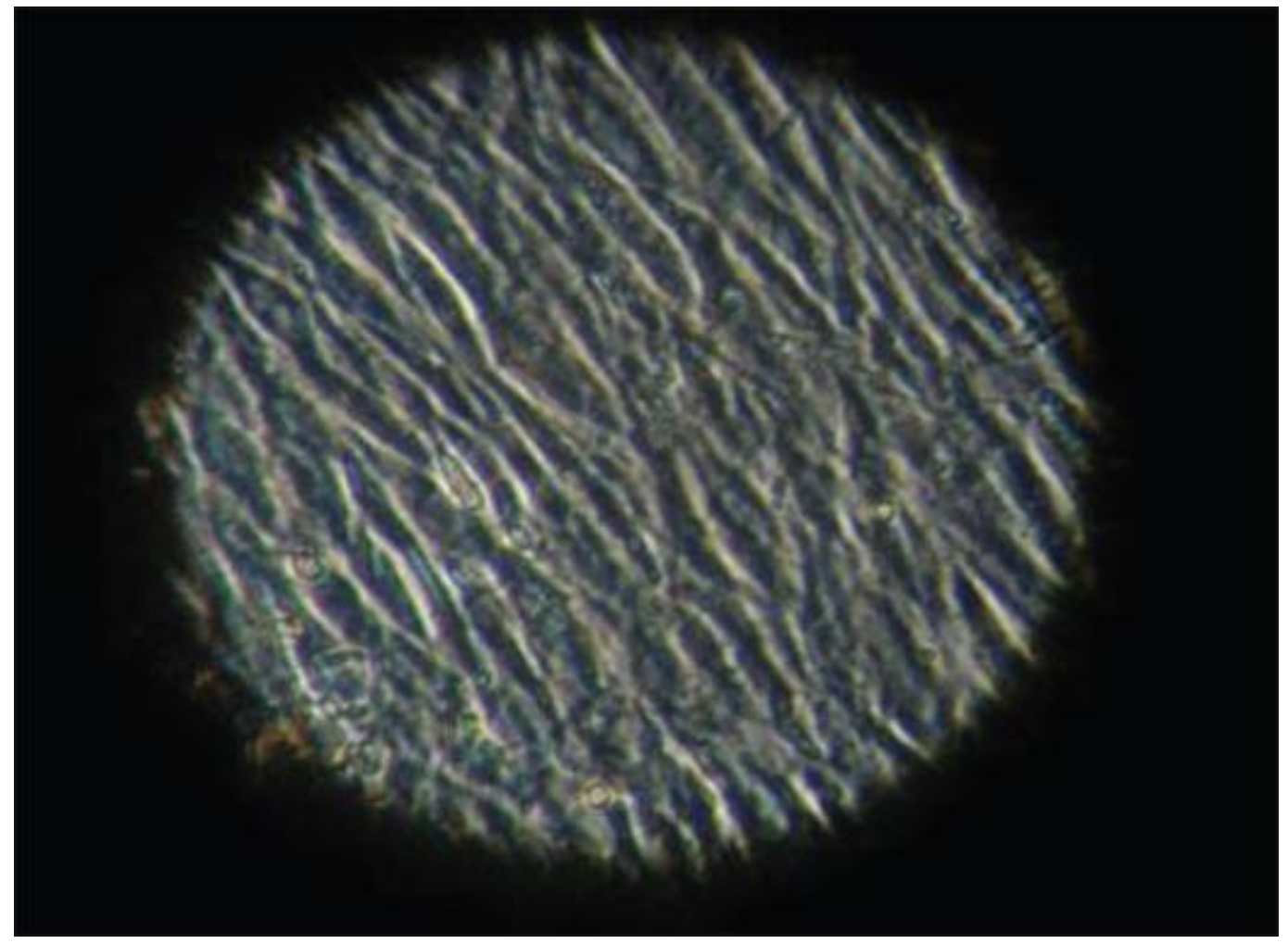

Figure 5: Monolayers of fibroblasts densely packed in 3D culture and generated by REGEN PRP 


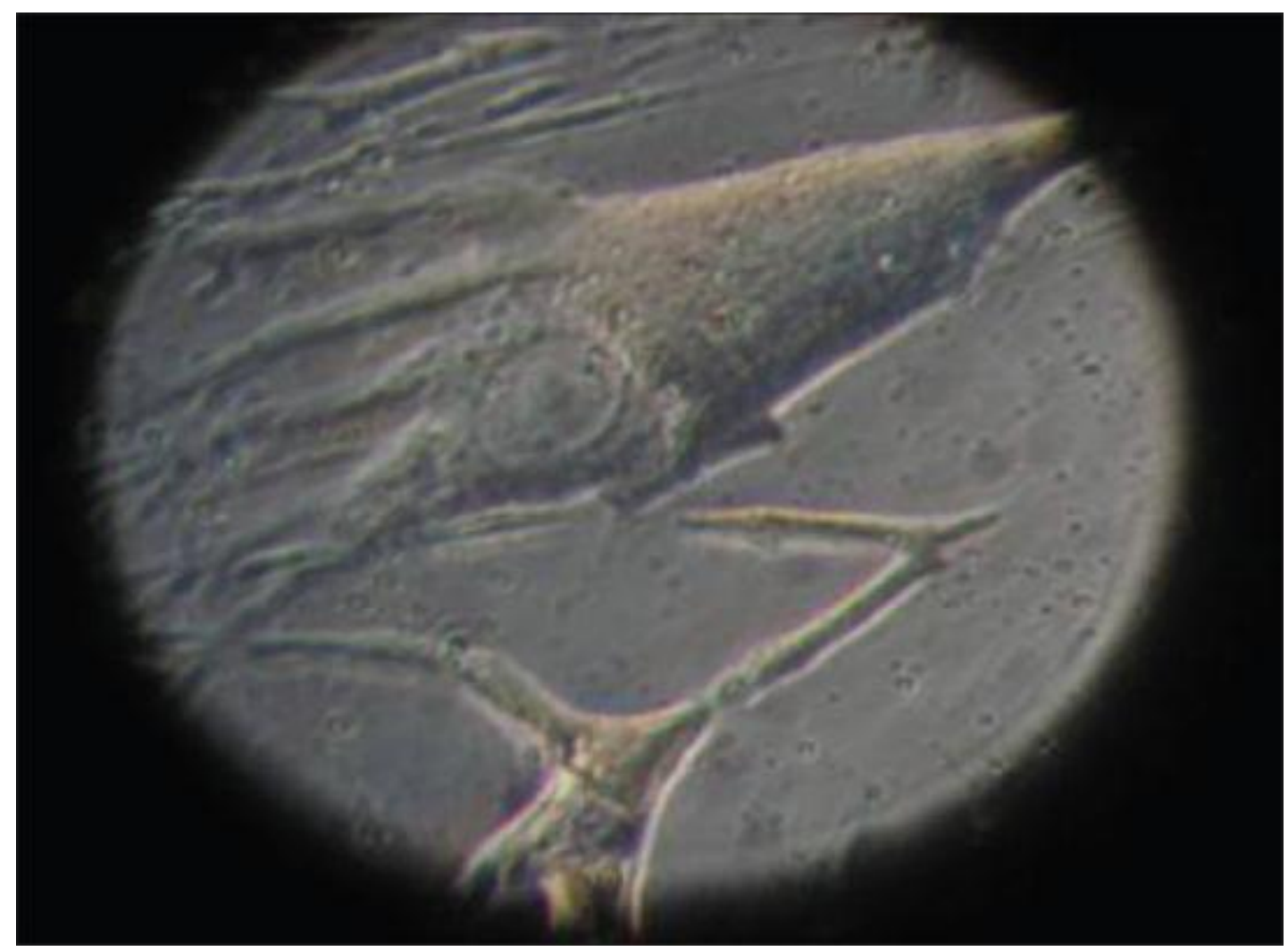

Figure 6: Tissue culture with PRP enriched medium showing a myofibroblast and adjacent fibroblast. Tissue markers for myofibroblasts include actin and vimentin

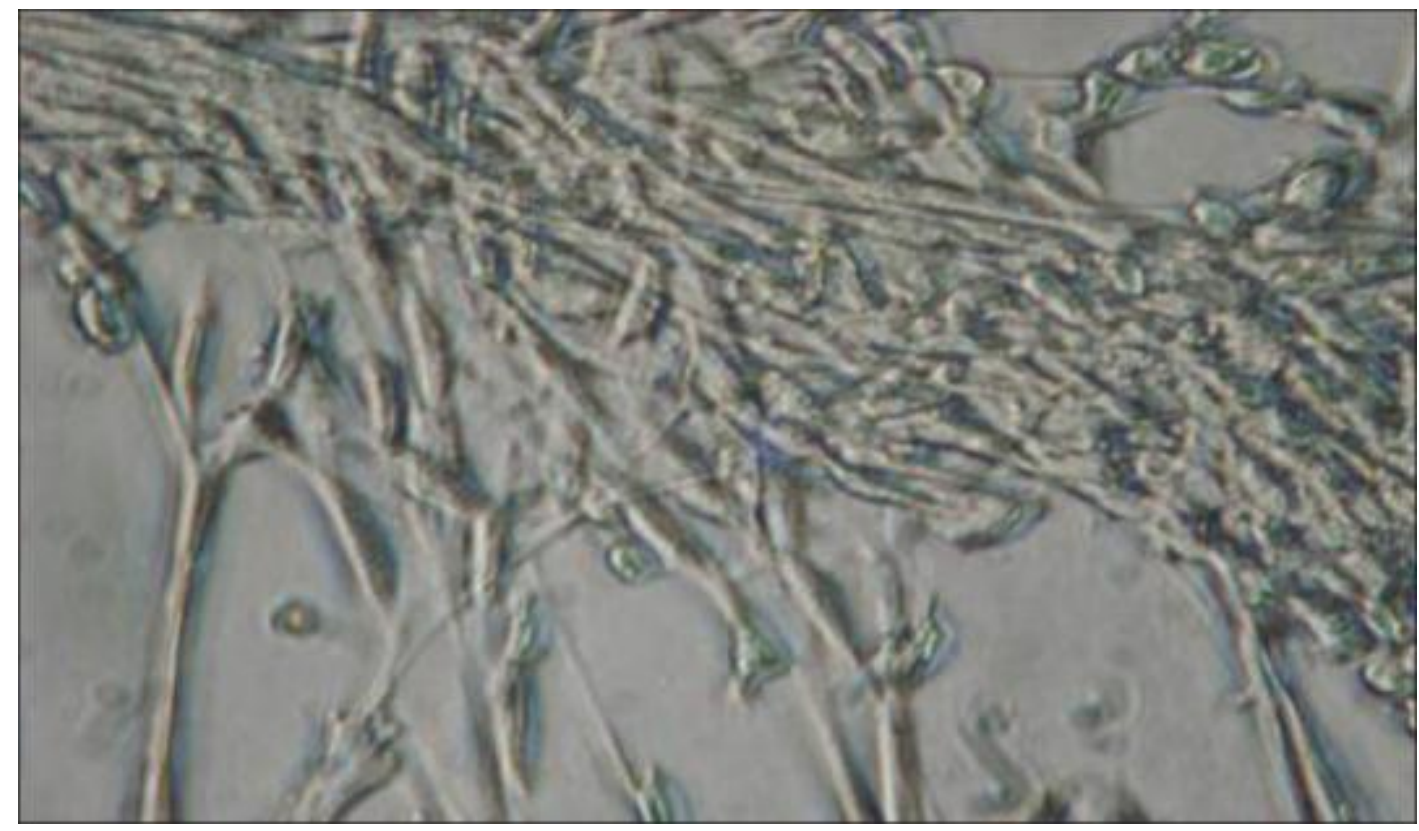

Figure 7: Fibroblasts in TC showing locomotion and crawling of fibroblasts in PRP enriched medium 


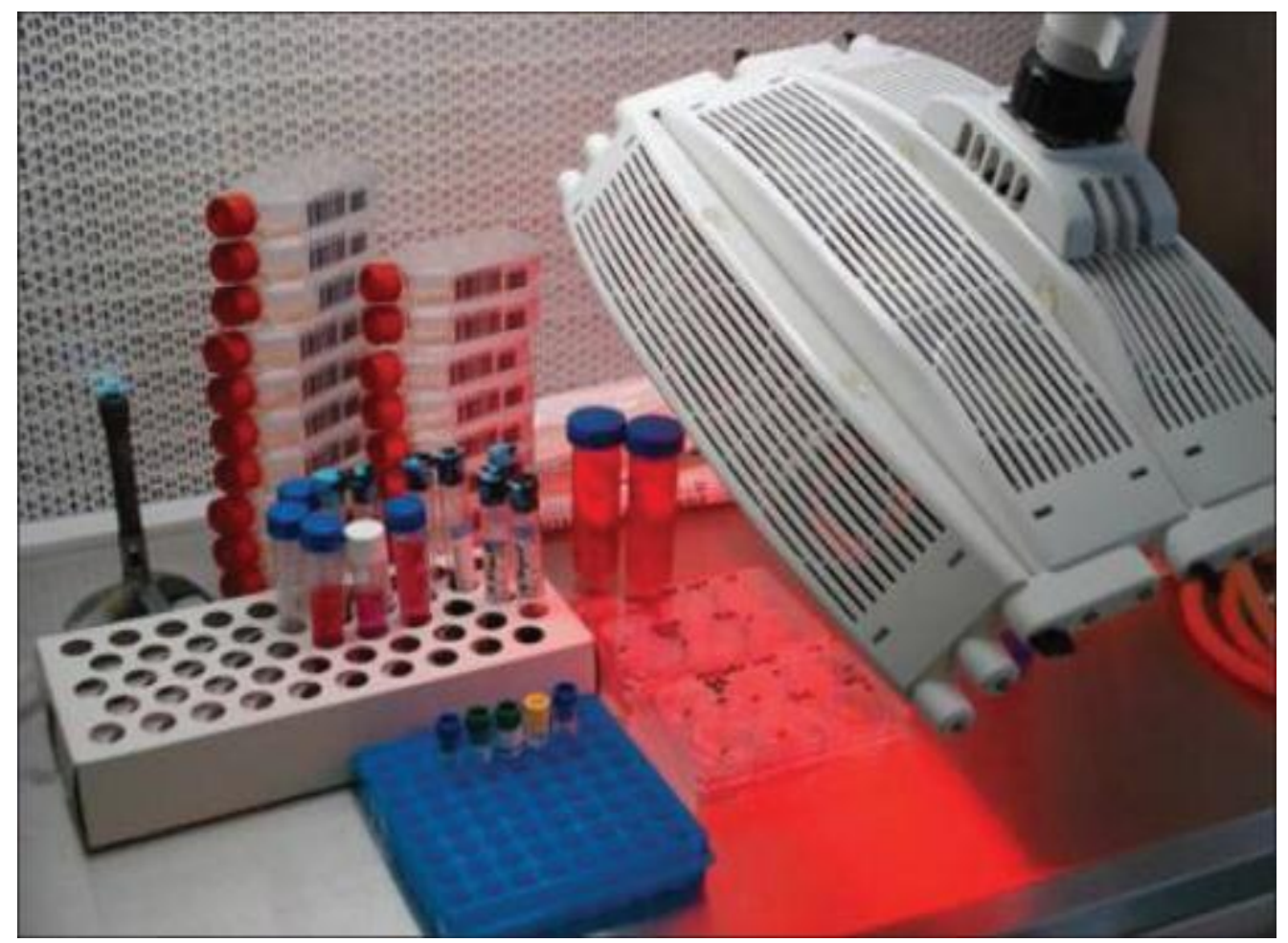

Figure 8: Stimulation of fibroblasts in tissue culture ex vivo, with red LED (633 nm)

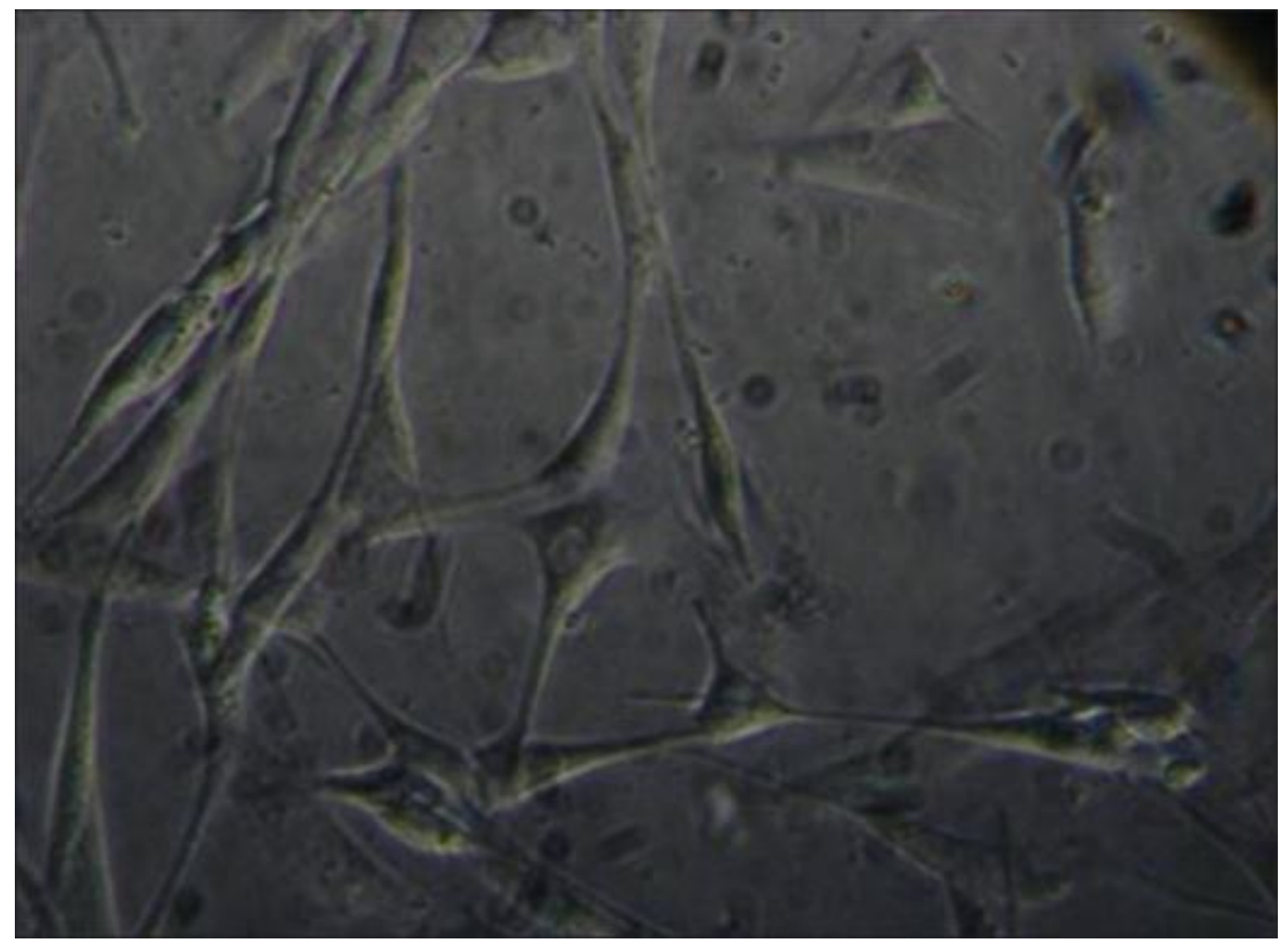

Figure 9: PRP generated 3D scaffold of human fi broblasts that are critical for the formation of the ECM. Note branching of the fibroblasts and cell-cell interaction 


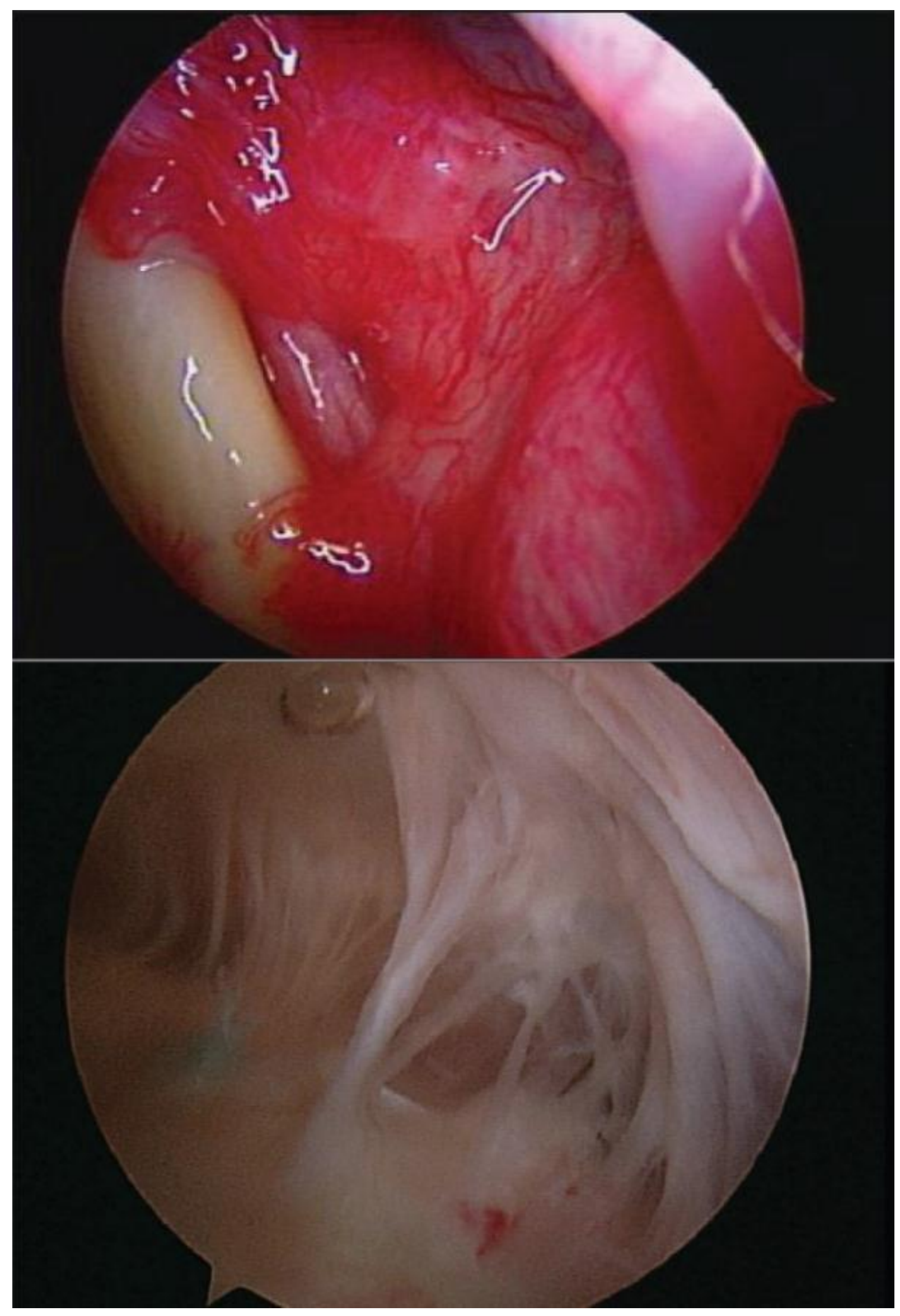

Figure 10A: Arthroscopic visualization of the rotator interval in a "frozen shoulder" Figure 10B: Arthroscopic visualization of the sub-deltoid space adhesions in a "captured shoulder" 


\section{Tables}

\begin{tabular}{l} 
Shape: Flattened, irregular, branching processes(fusiform or spindle \\
-shaped). \\
Cytoplasm: Clear, fine granules and may display fat droplets \\
Fibroblasts: Cytoplasm is branched, abundant and basophilic \\
Fibrocyte: (inactive cells). Cytoplasm is sparse and almost absent \\
on light microscopy. \\
Nucleus: Vesicular, oval or spheroidal-shaped, centrally placed, \\
fine chromatin granules, euchromatic staining and one or more \\
prominent nucleoli. \\
Cytocavitary network: Abundant mitochondria, RER and Golgi \\
apparatus \\
Associations: Production of collagen, ECM and proteoglycans \\
Transformation: Fibroblasts can differentiate into bone cells, \\
cartilage cells, fat cells and smooth muscle cells \\
Blood vessel association: Pericytes (these can differentiate into \\
fibroblasts) \\
Locomotion: Growth factors such as platelet-derived factors can \\
attract fibroblasts to regions of injury \\
Other features: Not all fibroblasts in different regions have the same \\
potential or capabilities. There is a possibility that different fibroblast \\
lineages exist. \\
Stages of activities: May be active or quiescent (fibrocytic) \\
\hline
\end{tabular}

Table 1: Classic histological features of fi broblasts: After Gray's anatomy[1,2] 


\section{Intracellular activity: Protein secreting cell[6]}

- Alpha chain, m RNA (in nucleus)

- Synthesis of alpha chains of preprocollagen in RER (hydroxylation of lysine and proline, glycosylation and disulphide bond formation ${ }^{[6]}$

- Hydroxylation of prolyl residues in the EPR

- Soluble galactosyl prolyl and lysyl residues to specific hydroxyl lysyl residues

- Procollagen molecule assembly

- Identification of registration peptides

- Transfer vesicles facilitate movement of procollagen to the Golgi complex from the RER

- Packaging of soluble procollagen in secretory vesicles of the Golgi apparatus

- Via secretory vesicles soluble procollagen molecules are transferred to the cell surface

\section{Extracellular enviroment}

- Discharge of procollagen molecules to ECM. Procollagen transformed to insoluble tropocollagen, which aggregate to form collagen fibrils

- Fibrillar structure is further reinforced by cross links of tropocollagen

- Synthesis of reticular and elastic fibres, glycosaminoglycans, proteoglycans and glycoproteins of the extracellular matrix $(\mathrm{ECM})^{[3,6]}$

- Production of growth factors (influence cell growth and differentiation ${ }^{[3]}$

- Transformation into myofibroblasts during wound healing (demonstrates features of both fibroblast and smooth cells; contain actin microfilaments and myosin; responsible for wound contraction during wound healing and closure. ${ }^{[6]}$

- Production of collagen is reflected in Table 2 and pathological conditions related to fibroblast dysfunction are reflected in Table 3.

Table 2: Molecular process of collagen synthesis by fibroblasts: After Kessel, Junqueira and Carneiro[3] 


- $\quad$ Heart: Cardiac myofibroblast expression (fibrosis $)^{[7]}$
- Inguinal hernia: Anomalies in collagen ratio and elastic fibers ${ }^{[8]}$
fibrosis ${ }^{[9]}$
- Solar or photo-aged face, neck and hands. Also includes the
chronological aged face.
- Scleroderma (systemic sclerosis)
- Inteloids: IL-6 derangement
Chronic ischaemic heart disease with remodelled heart (post-
myocardial infarction)
Retroperitoneal fibrosis

Table 3: Pathological disease processes related to abnormal or dysfunctional fibroblast and myofibroblast morphology and growth characteristics

\section{References}

1. Davies DV, Davies F. Gray's anatomy. Descriptive and applied. Longman's: London; 1962. p. 14-5.

2. Kessel RG. Basic medical histology. Biology of cells, tissues and organs. Oxford University Press: Oxford; 2003. p. 114-6.

3. Junqueira LC, Carneiro J. Basic histology. Text and Atlas. Lange: New York; 2003. p. 95125.

4. Dorland's illustrative medical dictionary. $30^{\text {th }}$ ed. Saunders: Philadelphia; 2003. p. 695.

5. Sadler TW. Langman's medical embryology. $9^{\text {th }}$ ed. Lippincott, Williams and Wilkins: Philadelphia; 2004. p. 90-1.

6. Kierszenbaum AL. Histology and cell biology. An introduction to pathology. Mosby: St Louis; 2002. p. 106-8.

7. Poobalarachi F, Baicu R, Bradshaw AD. Cardiac myofibroblasts differentiated in 3D culture exhibit distinct changes in collagen 1 production, processing and matrix deposition. Am J Physiol Heart Circ Physiol 2006;291:H2924-32.

8. Ozdogan M, Yildiz F, Gurer A, Orhun S, Kulacoglu H, Aydin R. Changes in collagen and elastic fiber contents of the skin, rectus sheath, transversalis fascia and peritoneum in primary inguinal hernia patients. Bratisl Lek Listy 2006;107:235-8.

9. Eyden B. Electron microscopy in the study of myofibroblastic lesions. Semin Diagn Pathol 2003;20:13-24.

10. Oda D, Gown AM, Vande Berg JS, Stern R. The fibroblast-like nature of myofibroblasts. Exp Mol Pathol 1998;49:316-29.

11. Shin D, Minn KW. The effect of myofibroblasts on contracture of hypertrophic scar. Plast Reconstr Surg 2004;113:633-40.

12. Badid C, Mounier N, Costa AM, Desmouliare A. Role of myofibroblasts during normal tissue repair and excessive scarring: Interest of their assessment in nephropathies. Histol Histpathol 2000;15:269-80.

13. Powell DW, Mifflin RC, Valentich JD, Crow SE, Saada JL, West AB, et al . Paracrine cells important in health and disease. Am J Physiol 1999;277:C1-9. 
14. Pardo A, Selman M. Molecular mechanisms of pulmonary fibrosis. Front Biosci 2002;17:d1743-61.

15. Desmouliare A. Factors influencing myofibroblast differentiation during wound healing and fibrosis. Cell Biol Int 1995;19:471-6.

16. Hinz B, Gabbiani G. Cell-matrix and cell-cell contacts of myofibroblasts: Role in connective tissue remodeling. Thromb Haemost 2003;90:993-1002.

17. Desmouliare A, Chaponnier C, Gabbiani G. Tissue repair, contraction and the myofibroblast. Wound Repair Regen 2005;13:7-12.

18. Hinz B, Phan SH, Thanickal VJ, Galli A, Bochaton-Piallot ML, Gabbiani G. The myofibroblast: One function, multiple origins. Am J Pathol 2007;170:1807-16.

19. Kumar V, Cotran RS, Robbins SL. Robbins basic pathology. $7^{\text {th }}$ ed. Saunders: Philadelphia; 2003. p. 74-5.

20. Bahar NA, Bamer B, Tredget EE, Ghahary A. Dermal fibroblasts from different layers of human skin are heterogeneous in expression of collagenase and types I and III procollagen mRNA. Wound Repair Regen 2004;12:175-82.

21. Marx RE, Garg AK. Dental and Craniofacial applications of platelet-rich plasma. Quintessence Books: Chicago; 2005. p. 1-154.

22. Eppley BL, Pietrzak Ws, Blanton W. Platelet-rich plasma: A review of biology and applications in plastic surgery. Plast Reconstr Surg 2006;118:147e-59e.

23. Pietrzak WS, Eppley BL. Platelet-rich plasma. Biology and new technology. J Craniofac Surg 2005;16:1043-54.

24. Du Toit DF, Kleintjes WG, Otto MJ, Mazyala EJ, Page BJ. Soft and Hard tissue-augmentation with platelet rich plasma: Tissue culture dynamics, regeneration and molecular biology perspective. Int J Shoulder Surg 2007;1:64-73.

25. Trelles Ma, Allones I. Red-light emitting diode (LED) therapy accelerates wound healing postblepharoplasty and periocular laser ablative resurfacing. J Cosmet Laser Ther 2006;8:3942.

26. Albert's B, Johnson A, Lewis J, Raff M, Roberts K, Walter P. Molecular Biology of the Cell. $4^{\text {th }}$ ed. Garland Science: New York; 2002. p. 1019-20.

27. Houveld N, Abrahamse $\mathrm{H}$. In vitro exposure of wounded diabetic fibroblast cells to a heliumneon laser at 5 and $16 \mathrm{~J} / \mathrm{cm}^{2}$. Photomed Laser Surg 2007;25:78-84.

28. Hawkins $\mathrm{DH}, \mathrm{Abrahamse} \mathrm{H}$. The role of laser fluence in cell viability, proliferation, membrane integrity of wounded human skin fibroblasts following helium-neon laser irradiation. Lasers Surg Med 2006;38:74-83.

29. Hawkins DH, Abrahamse H. Effect of multiple exposures of low level laser therapy on the cellular responses of wounded human skin fibroblasts. Photomed Laser Surg 2006;24:70514.

30. Smith SP, Devaraj VS, Bunker TD. The association between frozen shoulder and Dupuytren's disease. J Shoulder Elbow Surg 2001;10:149-51.

31. Mormino MA, Gross RM, Mc Carthy JA. Captured shoulder: A complication of rotator cuff surgery. Arthroscopy 1996;12:457-61.

32. Berghs B, Sole-Molins X, Bunker TD. Arthroscopic release of adhesive capsulitis. J Shoulder Elbow Surg 2004;13:180-5.

33. Omari A, Bunker TD. Open surgical release for frozen shoulder: Surgical findings and results of the release. J Shoulder Elbow Surg 2001;10:353-7.

34. Gamradt S, Rodeo S, Warren RF. Platelet rich plasma in rotator cuff repair. Tech Orthop 2007;22:26-33.

35. Pietrzak WS, An YH, Kang QK, Demos HA, Ehrens KH. Platelet-Rich and Platelet poor plasma: Development of an animal model to evaluate hemostatic efficacy. J Craniofac Surg 2007;18:559-67. 